\title{
Braces for idiopathic scoliosis in adolescents. A cochrane review
}

\author{
Stefano Negrini ${ }^{*}$, Silvia Minozzi, Josette Bettany-Saltikov, Fabio Zaina, Nachiappan Chockalingam, \\ Theodoros B Grivas, Tomasz Kotwicki, Toru Maruyama, Michele Romano, Elias S Vasiliadis \\ From 7th International Conference on Conservative Management of Spinal Deformities \\ Montreal, Canada. 20-22 May 2010
}

\section{Introduction}

Adolescent Idiopathic Scoliosis (AIS) is a three-dimensional deformity of the spine. While AIS can progress during growth and cause a surface deformity, it is usually not symptomatic. However, in adulthood, if the final spinal curvature surpasses a certain critical threshold, the risk of health problems and curve progression is increased. Braces are traditionally recommended to stop curvature progression in some countries and criticized in others. They generally need to be worn full time, with treatment extending over years. Our pur pose was to evaluate the efficacy of bracing in adolescent patients with AIS.

\section{Materials and methods}

The following databases (up to July 2008) were searched with no language limitations: the Cochrane Central Register of Controlled Trials, MEDLINE (from January 1966), EMBASE (from January 1980), CINHAL (from January 1982) and reference lists of articles. An extensive handsearch of the grey literature was also conducted. Randomised controlled trials and prospective cohort studies comparing braces with no treatment, other treatment, surgery, and different types of braces.

Two review authors independently assessed trial quality and extracted data.

\section{Results}

We included two studies. There was very low quality evidence from one prospective cohort study with 286 girls that a brace curbed curve progression at the end of growth (success rate $74 \%$ (95\% CI: $52 \%$ to $84 \%)$ ), better than observation (success rate $34 \%$ (95\% CI:16\% to
49\%)) and electrical stimulation (success rate 33\% (95\% CI:12\% to $60 \%)$ ). There is low quality evidence from one RCT with 43 girls that a rigid brace is more successful than an elastic one (SpineCor) at curbing curve progression when measured in Cobb degrees, but there were no significant differences between the two groups in the subjective perception of daily difficulties associated with wearing the brace.

\section{Conclusion}

There is very low quality evidence in favour of using braces, making generalization very difficult. Further research could change the actual results and our confidence in them; in the meantime, patients' choices should be informed by multidisciplinary discussion. Future research should focus on short and long-term patient-centred outcomes, in addition to measures such as Cobb angles. RCTs and prospective cohort studies should follow both the Scoliosis Resarch Society (SRS) and Society on Scoliosis Orthopaedic and Rehabilitation Treatment (SOSORT) criteria for bracing studies.

Published: 10 September 2010

doi:10.1186/1748-7161-5-S1-058

Cite this article as: Negrini et al: Braces for idiopathic scoliosis in adolescents. A cochrane review. Scoliosis 2010 5(Suppl 1):O58.

ISICO (Italian Scientific Spine Institute), Milan, Italy

Full list of author information is available at the end of the article 